Meta

Journal des traducteurs

Translators' Journal

\title{
La traduction médicale doit-elle être réservée aux seuls traducteurs-médecins ? Quelques réflexions
}

\section{Daniel Gile}

Volume 31, numéro 1, mars 1986

Traduction et terminologie médicale

Medical Translation and Terminology

URI : https://id.erudit.org/iderudit/002895ar

DOI : https://doi.org/10.7202/002895ar

Aller au sommaire du numéro

Éditeur(s)

Les Presses de l'Université de Montréal

ISSN

0026-0452 (imprimé)

1492-1421 (numérique)

Découvrir la revue

Citer cet article

Gile, D. (1986). La traduction médicale doit-elle être réservée aux seuls traducteurs-médecins ? Quelques réflexions. Meta, 31(1), 26-30.

https://doi.org/10.7202/002895ar d'utilisation que vous pouvez consulter en ligne. 


\section{LA TRADUCTION MÉDICALE DOIT-ELLE ÊTRE RÉSERVÉE AUX SEULS TRADUCTEURS-MÉDECINS ? QUELQUES RÉFLEXIONS}

DANIEL GILE

\section{INTRODUCTION}

Dans le secteur médical plus que dans beaucoup d'autres secteurs scientifiques et techniques, les donneurs d'ouvrage exigent souvent que les traductions soient faites par des spécialistes, en l'occurrence des médecins, voire des médecins spécialistes qui se limiteraient à leurs propres spécialités ; les non-spécialistes, et a fortiori les non médecins sont jugés incapables de réaliser des traductions correctes.

Le point de vue défendu dans le présent article se fonde sur la constatation empirique de l'identité qualitative des problèmes de la traduction dans une très large gamme de sujets scientifiques et techniques. Il existe bien des variations quantitatives selon le sujet, mais les variations dues à d'autres variables, et notamment à la qualité de l'écriture du texte original et à la structure des langues concernées sont en général bien plus importantes, et les problèmes posés sont essentiellement les mêrnes.

Le présent article explique les problèmes fondamentaux de la traduction scientifique et technique en général, aborde plus particulièrement le cas de la traduction médicale, et décrit une méthode de travail que suit l'auteur dans ses traductions médicales dans un but d'optimisation de la qualité du travail.

\section{LES PROBLÈMES DE LA TRADUCTION SCIENTIFIQUE ET TECHNIQUE} tants :

La traduction scientifique et technique se caractérise par deux éléments impor-

- elle se fond sur des contenus cognitifs spécialisés que ne possèdent que les spécialistes - elle véhicule essentiellement des messages informatifs plutôt qu'affectifs ou esthétiques.

Il semble maintenant acquis que dans la traduction humaine, l'acte traduisant n'est pas une opération de transcodage linguistique, mais l'enchaînement d'une phase d'assimilation du message original et d'une phase de réexpression dans la langue d'arrivée (voir par exemple Lederer 1973).

Vu sous cet angle, la traduction scientifique et technique pose des problèmes de compréhension aussi bien que des problèmes d'expression.

On sait que la compréhension d'un texte par le lecteur est la résultante de deux composantes distinctes : l'analyse des structures linguistiques, qui permet d'avoir une idée de la nature des relations fonctionnelles et logiques entre des mots et groupes de mots, et l'interprétation des structures linguistiques et des mots en fonction d'une connaissance extra-linguistique. En effet, tout énoncé linguistique pris isolément est toujours ambigu en un ou plusieurs endroits, et seule la connaissance extra-linguistique permet de résoudre cette ambiguïté (voir Pergnier 1973 : 31). En fait, plus les connais- 
sances extra-linguistiques du lecteur sont importantes, plus l'énoncé linguistique peut être interprété avec précision.

Dans l'énoncé : La croissance de régions offre l'avantage de conduire à des contours fermés ${ }^{1}$, le lecteur non spécialisé comprend d'après la structure linguistique et ses connaissances générales qu'un élément 'A' (la croissance de régions) présente l'avantage ' B' (de conduire à des contours fermés), mais il ne sait pas interpréter ' A' et 'B'. S'il a lu un article de vulgarisation sur les images électroniques, il sait peut-être que ' $A$ ' est une méthode d'analyse de l'image, et que ' $B$ ' présente une caractéristique géométrique de l'image (ce qui lui permettra d'ailleurs de déduire que la caractéristique contours fermés est une bonne chose dans ce contexte, et de se servir de cet élément d'information nouveau par la suite). Seul le vrai spécialiste a les connaissances nécessaires pour dégager de l'énoncé le message de l'auteur dans toute sa netteté.

On notera au passage que les deux termes techniques très spécialisés, croissance de régions et contours fermés, sont composés de mots très courants, exemple qui illustre bien la polysémie obligatoire de la langue ; avec un nombre de mots très limité, il faut exprimer un nombre de concepts sans cesse croissant, et la langue doit donc souvent attribuer des sens nouveaux à des mots existants ou créer des entités nouvelles formées de deux ou plusieurs mots existants.

Étant donné l'infériorité cognitive du traducteur par rapport à l'auteur du texte spécialisé qu'il traduit, sa compréhension de l'énoncé est inférieure à celle du spécialiste, ce qui peut se manifester par des termes incompris ou mal compris, ainsi que par l'incapacité d'interpréter ou une interprétation erronée de certains liens logiques ou fonctionnels que l'énoncé linguistique ne précise pas de manière univoque (voir exemples plus loin).

En outre, le traducteur qui ne possède pas la matière dans laquelle se situe le texte ne possède en règle générale que fragmentairement le vocabulaire et les structures linguistiques qui y correspondent, ce qui pose des problèmes de réexpression lors de la deuxième phase de la traduction.

\section{LA TRADUCTION MÉDICALE}

La traduction médicale répond bien à la définition de la traduction scientifique et technique formulée au début de la section précédente. La médecine est en effet un faisceau de disciplines très spécialisées qui se fondent, dès le niveau du médecin généraliste, sur un vaste ensemble de concepts constitués (éléments de savoir et de savoir-faire considérés comme discrets et ayant des noms). La chose est d'ailleurs frappante sur le plan intuitif, quand on compare le nombre d'années d'études dans le cursus de médecine à la longueur des autres études spécialisées, d'autant plus que dans les études de médecine, une importante proportion d'heures est consacrée à l'acquisition d'un savoir, par opposition à l'acquisition d'un savoir-faire fondé sur un savoir acquis précédemment. En outre, la traduction médicale est bien informative, même si le 'méta-message' des traductions demandées par les laboratoires pharmaceutiques est souvent le même : ' prescrivez tel produit, c'est le meilleur !'

En tant que domaine scientifique, la médecine pose pour les traducteurs les problèmes de compréhension et de réexpression évoqués plus haut, peut-être davantage encore que les autres spécialités scientifiques et techniques (une telle comparaison n'a toutefois qu'une valeur statistique).

Pour le traducteur qui n'est pas médecin, le vocabulaire médical est une source apparemment intarissable. L'auteur du présent article a lu, traduit ou interprété en conférence plusieurs milliers de pages de textes médicaux ; pourtant, chaque nouveau texte présente un vocabulaire nouveau, et la proportion de mots nouveaux par page de texte telle qu'apparaissant dans les lexiques constitués à l'occasion de chaque conférence et de 
chaque traduction est nettement supérieure à la proportion de mots nouveaux relevés dans les autres spécialités dans lesquelles travaille l'auteur.

Cette prolifération dans le lexique médical destiné aux seuls médecins a donné lieu à la publication de nombreux dictionnaires encyclopédiques, eux aussi destinés aux médecins. C'est pourquoi on y trouve les concepts fondamentaux, mais les mots composés par ces concepts fondamentaux et des modificateurs, que les médecins comprennent aisément grâce à leur savoir spécialisé, n'y figurent que partiellement. Or, pour le profane, ces concepts ne sont pas toujours compréhensibles, et quand ils le sont, c'est la connaissance des termes idiomatiques équivalents en langue d'arrivée qui manque souvent : comment le profane arrivera-t-il de 'level of $G A B A$ in the brain' à ' taux intra-cérébral de GABA', de 'GABA mediated inhibitory systems' à 'systèmes inhibiteurs GABAergiques', de 'spontaneous firing rates' à 'fréquence des décharges spontanées' ?

Outre ces problèmes lexicaux, se posent des problèmes d'interprétation relationnelle : sans des connaissances spécialisées, comment interpréter 'potentiation of postsynaptic GABA responses' par ' potentialisation de la réponse postsynaptique au GABA', et ' $G A B A$ potentiation' par ' potentialisation du GABA', ces deux expressions intervenant dans le même paragraphe?

Le problème se trouve aggravé par le fait que la publication d'articles ayant la valeur qu'elle a dans le milieu médical hospitalier et de recherche, les médecins chercheurs écrivent beaucoup, dans un style dont la clarté laisse parfois à désirer, et souvent dans une langue qu'ils ne maîtrisent que très imparfaitement.

Dans ces conditions, on admet sans peine que le déchiffrage et la réexpression des textes médicaux demandent en général des connaissances assez poussées, et on comprend pourquoi certains donneurs d'ouvrage pensent que seuls les médecins sont capables de faire correctement des traductions médicales.

Et pourtant, on peut se demander s'il suffit d'être médecin et de connaître la langue de départ pour traduire des textes médicaux vers sa langue maternelle. On peut aussi se poser la question de savoir s'il n'y a pas moyen pour un traducteur qui ne serait pas médecin de réunir les éléments nécessaires pour réaliser de bonne traductions médicales.

Il convient de se rappeler à ce propos que dans le produit fini, la part de la réexpression est aussi importante que la part de la compréhension du texte original. Dans ce deuxième stade de l'opération, le traducteur doit rédiger un texte clair et lisible, qui soit fidèle à l'original sans être contaminé par la langue de départ. L'expérience montre qu'en général, seuls les traducteurs professionnels ont la compétence requise ; les amateurs, $\mathrm{y}$ compris les traducteurs occasionnels qui maîtrisent très bien la langue d'arrivée en d'autres circonstances, ont tendance à faire du mot-à-mot, ce qui donne des textes inélégants au mieux et parfois incompréhensibles. En effet, ces traducteurs non professionnels craignent de s'écarter du sens en s'écartant des structures linguistiques du texte original (voir à ce propos une explication fondamentale dans Gile 1982).

Le bon traducteur est un bon rédacteur technique, et il sait traduire ; le bon médecin n'est pas forcément un bon rédacteur technique, et il est rare qu'il sache traduire.

Dans ces conditions, la solution la plus logique consiste à réunir les deux compétences, ce qui se traduit en France, et probablement dans la plupart des pays occidentaux, par trois formules de base :

\section{Le médecin-traducteur}

Il existe des médecins devenus traducteurs, ainsi que des médecins qui suivent les deux carrières en parallèle. Ces professionnels sont rares et précieux. En raison de leur 
double compétence, les problèmes de la traduction médicale évoqués plus haut ne se posent pas dans leur travail.

\section{Le médecin-traducteur occasionnel}

À côté du médecin-traducteur professionnel existent de nombreux médecins-" faux traducteurs", pour qui la traduction est une activité occasionnelle complémentaire. En général, ces médecins ne maîtrisent pas la méthode, et ils ne sont pas toujours bons rédacteurs. Pour la plupart, ces traducteurs occasionnels travaillent pour des intermédiaires, "cabinets de traduction " ou assimilés, qui sont obligés de réviser en profondeur le travail rendu pour en faire un produit fini convenable. Or, la meilleure révision ne peut transformer une traduction médiocre en un bon texte, et la révision est souvent un travail aussi ardu que la traduction même. La formule du médecin-« faux traducteur » nous semble peu satisfaisante pour le destinataire, qui reçoit un produit souvent médiocre, et encore moins satisfaisante pour le traducteur luimême, qui est très mal rémunéré par rapport à un traducteur travaillant sans intermédiaire. Il se forme d'ailleurs un cercle vicieux, car un traducteur mal rémunéré aura du mal à " rentabiliser " son activité s'il ne va pas vite, et une vitesse excessive nuit à la qualité. On constate d'ailleurs en s'entretenant avec ces traducteurs occasionnels que les conditions dans lesquelles ils travaillent les "démotivent". Le phénomène est banal dans toute activité professionnelle, mais il mérite d'être souligné une nouvelle fois dans le contexte de la traduction, où la présence ou l'absence de motivation peut modifier très sensiblement la qualité d'un texte rendu, même chez des traducteurs dotés d'une conscience professionnelle supérieure à la moyenne.

\section{La collaboration médecins-traducteurs}

Une autre formule consiste pour le traducteur à rechercher l'appui des médecins pour les problèmes de compréhension et de réexpression. En général, le traducteur lit le texte original, repère les problèmes, et demande à son correspondant médecin ce que veut dire telle phrase ou telle expression, et comment réexprimer tel terme dans la langue d'arrivée. Dans la mesure où les questions ne portent que sur les termes que les traducteurs ne trouvent pas dans les dictionnaires, et compte tenu de la fiabilité toujours incertaine des dictionnaires, surtout quand leurs utilisateurs ne sont pas médecins, on trouve souvent dans les traductions des professionnels des maladresses ou des contresens dus à des connaissances insuffisantes.

\section{UNE MÉTHODE PRATIQUE}

Pendant plusieurs années, l'auteur du présent article a travaillé selon la $3^{\mathrm{e}}$ formule ci-dessus, en comptant sur les réviseurs médecins pour corriger d'éventuelles maladresses et erreurs. Â la suite de la rencontre d'un médecin-traducteur occasionnel, il a commencé il y a quelque temps à travailler selon une formule nouvelle.

Contrairement à la méthode classique, dans laquelle c'est le traducteur qui assure la première phase de la préparation en lisant le texte et en repérant les problèmes, dans cette nouvelle méthode, le traducteur commence par envoyer le texte original au médecin. Celui-ci le lit et note systématiquement en français (langue maternelle du traducteur et du médecin et langue d'arrivée de la traduction) les termes techniques, ainsi que des explications sur des phrases qui lui paraissent ambiguës. Le traducteur n'a plus qu'à rédiger en se servant de la liste faite par le médecin.

L'intérêt méthodologique de ce système est évident, puisque la compétence rédactionnelle et méthodologique du traducteur professionnel est complétée par les connaissances spécialisées du médecin, et ce pour l'ensemble du texte, ce qui représente une amélioration qualitative considérable par rapport aux solutions partielles de la formule 
classique. En outre, il s'avère que le travail du médecin est rapide (4 à 8 pages de texte original ou davantage par heure) et que le traducteur fait des économies de temps considérables : en effet, il est rare que les recherches terminologiques demandent moins de $20 \%$ du temps de travail du traducteur; en fait, elles en représentent souvent plus de $50 \%$; dans ces conditions, le traducteur peut se permettre de rétrocéder au médecin une somme nettement supérieure à celle que celui-ci gagnerait en consacrant un temps équivalent à la traduction complète pour un intermédiaire, ce qui rend l'opération financièrement intéressante pour les deux parties.

\section{CONCLUSION}

Depuis qu'il a commencé à travailler selon cette nouvelle méthode dans le domaine médical, l'auteur en est devenu un partisan convaincu. Compte tenu de l'amélioration de la qualité du travail qu'elle implique, il refuse actuellement des traductions médicales quand les conditions ne sont pas réunies pour qu'elle puisse être utilisée.

La méthode présentée ici résulte de la constatation d'un fait que les traducteurs hésitent souvent à reconnaître : en dépit de l'existence des dictionnaires et autres sources terminologiques et documentaires, ils manquent parfois d'éléments de connaissance et ne peuvent faire leur travail dans les meilleures conditions. En faisant appel à des spécialistes-" faux traducteurs " qui ont ces connaissances, mais qui ne possèdent pas la compétence méthodologique et rédactionnelle nécessaire, on ne fait que déplacer les insuffisances sans nécessairement améliorer le résultat. C'est pourquoi nous pensons que, en l'absence de spécialistes ayant une véritable double compétence, la solution passe par le travail en équipes bidisciplinaires formées autour du traducteur. Cette formule permet à celui-ci de fournir un travail de bonne qualité dans des domaines spécialisés variés. Les sciences médicales en sont un bon exemple d'application.

\section{Notes}

1. Énoncé extrait d'un texte sur les images électroniques.

\section{BIBLIOGRAPHIE}

GILE, Daniel (1982) : "Fidélité et littéralité dans la traduction : une approche pédagogique ", Babel, XXVIII- 1 .

LEDERER, Marianne (1973) : "La traduction : transcoder ou réexprimer ? ", Ela, no 12, octobre-décembre. PERGNIER, Maurice (1973) : "Traduire et théorie linguistique ", Ela, no 12 , octobre-décembre. 